\title{
Proteomic identification of arginine-methylated proteins in colon cancer cells and comparison of messenger RNA expression between colorectal cancer and adjacent normal tissues
}

\author{
Yongchul Lim, Da Young Gang, Woo Yong Lee, Seong Hyeon Yun, Yong Beom Cho, Jung Wook Huh, \\ Yoon Ah Park, Hee Cheol Kim \\ Department of Surgery, Samsung Medical Center, Sungkyunkwan University School of Medicine, Seoul, Korea
}

Purpose: Identification of type I protein arginine methyltransferase (PRMT) substrates and their functional significance during tumorigenesis is becoming more important. The present study aimed to identify target substrates for type I PRMT using 2-dimensional (2D) gel electrophoresis (GE) and 2D Western blotting (WB).

Methods: Using immunoblot analysis, we compared the expression of type I PRMTs and endogenous levels of arginine methylation between the primary colorectal cancer (CRC) and adjacent noncancerous tissues paired from the same patient. To identify arginine-methylated proteins in HCT116 cells, we carried out 2D-GE and 2D-WB with a type I PRMT product-specific antibody (anti-dimethyl-arginine antibody, asymmetric [ASYM24]). Arginine-methylated protein spots were identified by mass spectrometry, and messenger RNA (mRNA) levels corresponding to the identified proteins were analyzed using National Center for Biotechnology Information (NCBI) microarray datasets between the primary CRC and noncancerous tissues.

Results: Type I PRMTs and methylarginine-containing proteins were highly maintained in CRC tissues compared to noncancerous tissues. We matched 142 spots using spot analysis software between a Coomassie blue (CBB)-stained 2D gel and 2D-WB, and we successfully identified 7 proteins that reacted with the ASYM24 antibody: CACYBP, GLOD4, MAPRE1, CCT7, TKT, CK8, and HSPA8. Among these proteins, the levels of 4 mRNAs including MAPRE1, CCT7, TKT, and HSPA8 in CRC tissues showed a statistically significant increase compared to noncancerous tissues from patients using the NCBI microarray datasets.

Conclusion: Our results indicate that the method shown here is useful in identifying arginine-methylated proteins, and significance of arginine modification in the proteins identified here should be further identified during CRC development.

Keywords: Colorectal neoplasms; Protein-arginine N-methyltransferases; N,N-dimethylarginine; Two-dimensional gel electrophoresis; Mass spectrometry

Received: Oct 21, 2020 - Revised: Jun 21, 2021 - Accepted: Jun 29, 2021 Correspondence to: Hee Cheol Kim, M.D.

Department of Surgery, Samsung Medical Center, 81 Irwon-ro, Gangnam-gu, Seoul 06351, Korea

Tel: +82-2-3410-1655, Fax: +82-2-3410-6980

E-mail: hckim@skku.edu

ORCID: https://orcid.org/0000-0002-1610-5363

(C) 2022 The Korean Society of Coloproctology

This is an open-access article distributed under the terms of the Creative Commons Attribution NonCommercial License (https://creativecommons.org/licenses/by-nc/4.0) which permits unrestricted noncommercial use, distribution, and reproduction in any medium, provided the original work is properly cited.

\section{INTRODUCTION}

Colorectal cancer (CRC) is the 3rd most deadly and the 4th most commonly diagnosed cancer in the world [1]. The development and progression of CRC involve multiple different molecular mechanisms including mitogen-activated protein kinase cascades downstream of epidermal growth factor receptor, Notch, PI3K/AKT pathway, transforming growth factor- $\beta$, and $\mathrm{Wnt} / \beta$-catenin signaling pathways [2]. In addition, mutations in various components of these signaling pathways have been linked to the development of CRC. Accordingly, there have been numerous efforts to target these 
signaling pathways to develop novel therapeutic approaches. However, as not all CRCs share similar driving mutations, it has been difficult to design a "catch-all" molecular therapy [3].

Protein arginine methylation is a common posttranslational modification that plays a critical role in a variety of intracellular events, such as DNA repair, signal transduction, protein-protein interaction, and transcriptional regulation $[4,5]$. The reaction is catalyzed by a class of enzymes called protein arginine methyltransferases (PRMTs). PRMTs are classified into 2 major groups, type I and type II, according to their end products. Both type I and II enzymes can generate monomethyl arginine (MMA) as an intermediate. Type I enzymes (PRMT1, 2, 3, 4, 6, and 8) produce asymmetric $N^{G}, N^{G}$-dimethylarginine (ADMA), whereas type II enzymes (PRMT5 and 9) generate symmetric $N^{G}, N^{G}$-dimethylarginine (SDMA) [4-6]. PRMT7 is a unique class III PRMT enzyme that only monomethylates arginine residue [7]. Recent studies have implicated protein arginine modification in the pathogenesis of various human diseases including cancer $[8,9]$, and PRMTs are emerging as potential therapeutic targets [10]. PRMT1 is significantly higher in colon cancer tissue compared with normal tissue, and it is associated with clinical and histological parameters such as nodal status and stage [11]. PRMT4/coactivator-associated arginine methyltransferase 1 is a positive modulator of $\mathrm{WNT} / \beta$ catenin-driven transcription and neoplastic transformation in CRC cells [12], and observations from clinical samples have shown that 75\% of CRCs overexpress PRMT4 [13]. In our recent study, patients with PRMT6-positive CRC by immunohistochemistry had shorter disease-free survival than those with PRMT6negative CRC in both univariate and multivariate analyses [14]. Consequently, identifying the location of methylarginine residues within PRMTs substrates and the functional significance of their modification during tumorigenesis is a critical issue.

The development of highly specific antibodies against MMA, ADMA, and SDMA in proteins has made it possible to enrich arginine-methylated peptides after trypsin digestion $[15,16]$. Using immunoenrichment of arginine-methylated peptides combined with mass spectrometry (MS)-based proteomics technology, almost 10,000 arginine-methylated sites have been identified in various biological sources [16-19]. Using this approach, we identified 455 MMA sites in 272 proteins and 314 ADMA sites in 155 proteins from CRC tissues acquired from patients [20]. In addition, we identified 147 SDMA sites in 94 proteins, and quantitative analysis comparing CRC and normal tissues revealed a significant increase in the symmetric dimethylation of 70 arginine sites in 46 proteins and a decrease in that of 4 arginine sites in 4 proteins [21]. However, this approach requires large amount of protein (10 mg per 1 type of arginine modification). Besides, unlike phospho-specific antibodies, currently developed antibodies against methyl-arginine have their preferential antigen motifs such as a glycine-arginine-rich region [22]. Therefore, the immunoenrichment approach for identifying methyl-arginine sites in proteins is highly dependent on the antibody used.
In the present study, we compared endogenous levels of ADMAcontaining proteins between $\mathrm{CRC}$ and matched normal tissues from patients using anti-dimethyl-arginine antibody, asymmetric (ASYM24) antibody which is a representative polyclonal antibody specific to ADMA [23] that is not used in immunoenrichment methods. Using 2-dimensional (2D) gel electrophoresis (GE) and 2D-Western blotting (WB) analysis with ASYM24 antibody followed by MS, we aimed to identify ADMA-containing proteins in HCT116 cells and compared the messenger RNA (mRNA) levels corresponding to the ADMA-containing proteins identified in HCT116 cells between CRC and noncancerous tissues from patients. We demonstrate that this approach is a useful tool for identifying methylarginine-containing proteins in clinical tissues as well as established cell lines.

\section{METHODS}

\section{Tissue samples from patients with colorectal cancer}

Patients diagnosed with primary CRC underwent radical surgery at the Samsung Medical Center in Seoul, Korea. We obtained prior patient consent as well as approval from the Institutional Review Board (No. 2017-05-065) to use clinical materials for research purposes. Eleven frozen CRC tumors and matched adjacent normal tissues were collected after surgery and the tissue samples were immediately frozen in liquid nitrogen. For protein extraction, 50-100 mg of tissue was homogenized using a TissueLyser (Qiagen, Hilden, Germany) in lysis buffer (7 $\mathrm{M}$ urea, $2 \mathrm{M}$ thiourea, 4\% [w/v] 3-[(3-cholamidopropyl) dimethyl-ammonio]1-propanesulfonate [Merck, Darmstadt, Germany], 0.5\% immobilized $\mathrm{pH}$ gradient buffer [GE Healthcare, Uppsala, Sweden], and 65-mM dithiothreitol [DTT], $1 \times$ protease and phosphatase inhibitors) and sonicated for 3 bursts of 30 seconds at $15 \mathrm{~W}$. The extracts were then centrifuged at $20,000 \times g$ at $4^{\circ} \mathrm{C}$ for 15 minutes and the supernatant was stored at $-80^{\circ} \mathrm{C}$ until use.

\section{Cell culture, MS023 treatment, and protein extraction}

Two human colon cancer cell lines, DLD1 and HCT116, were obtained from the American Type Culture Collection (Manassas, VA, USA). DLD1 and HCT116 cells were cultured in RPMI-1640 and McCoy's 5A media, respectively. All culture media were supplemented with $10 \%$ fetal bovine serum and $1 \%$ penicillin/streptomycin (Gibco, Grand Island, NY, USA). Cells were maintained at $37^{\circ} \mathrm{C}$ with $5 \% \mathrm{CO}_{2}$. Media were changed every 3 days. Cells were routinely subcultured when they reached $80 \%$ to $90 \%$ confluency. For MS023 treatment, both cell lines were seeded at a density of $1.0 \times 10^{6}$ cells in a 100 -mm dish. After 24 hours incubation, cells were treated with increasing concentrations of MS023 as indicated in Fig. 1 for 48 hours. Cells were harvested in radioimmunoprecipitation assay lysis buffer supplemented with protease and phosphatase inhibitors. Lysates were briefly sonicated and centrifuged at 13,000 revolutions per minute (rpm) for $15 \mathrm{~min}$ utes. The supernatant was then stored at $-80^{\circ} \mathrm{C}$ before use. 


\section{Coloproctology rongchul lim, et al.}
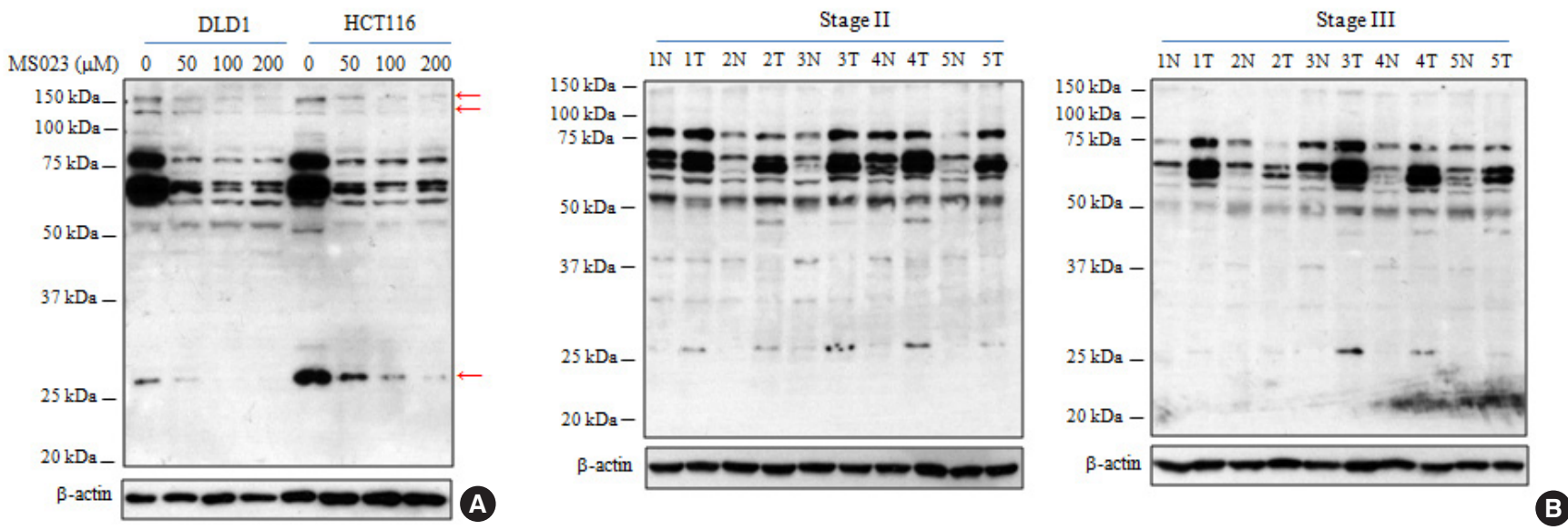

Fig. 1. The patterns of asymmetric dimethylarginine (ADMA)-containing proteins in colorectal cancer (CRC) cell lines and tissues from patients. (A) The endogenous level of ADMA-containing proteins was analyzed by Western blot. (B) Using the same experimental conditions shown in Fig. 2, asymmetric arginine dimethylation status in CRC tissues was compared by Western blot.

To extract cellular proteins for $2 \mathrm{D}-\mathrm{GE}$, cells at about $90 \%$ confluence were washed twice with phosphate-buffered saline (PBS), scraped on ice in PBS, collected by centrifugation, and then lysed in the rehydration buffer mentioned above. The lysate was sonicated for 5 seconds at $60 \%$ amplitude, and insoluble material was pelleted by centrifugation at $13,000 \mathrm{rpm}$ for 20 minutes. The supernatant was then stored at $-80^{\circ} \mathrm{C}$ before use. Protein concentration was determined by the Bradford assay.

\section{Two-dimensional electrophoresis and protein spot analysis} Isoelectric focusing (IEF) was carried out using IPG gel strips (pH 3-10 nonlinear, $13 \mathrm{~cm}$; GE Healthcare, Uppsala, Sweden) on the Multiphor II system (GE Healthcare). Samples containing $200 \mu \mathrm{g}$ of proteins were diluted with rehydration solution (GE Healthcare) to a final volume of $250 \mathrm{~mL}$ and applied to IPG tray followed by 15 hours of rehydration at $20^{\circ} \mathrm{C}$. Proteins were focused for a total of 85,000 Vh. After IEF, the strips were equilibrated in equilibration buffer (50-mM Tris- $\mathrm{HCl}, \mathrm{pH} 8.8,6 \mathrm{M}$ urea, 30\% glycerol, $2 \%[\mathrm{w} / \mathrm{v}$ ] sodium dodecyl sulfate [SDS], $20-\mathrm{mg} / \mathrm{mL}$ DTT, and $0.0125 \%$ bromophenol blue) for 15 minutes and then for an additional 15 minutes in the same solution except that DTT was replaced with $25 \mathrm{mg} / \mathrm{mL}$ iodoacetamide. SDS-polyacrylamide gel electrophoresis (PAGE) was used for the second separation in a Bio-Rad PROTEAN II xi gel system (Bio-Rad, Hercules, CA, USA). Total spot number and matching between the CBB-stained 2D gel and the immunoblot images were analyzed by PDQuest software (ver. 8.0, Bio-Rad).

\section{Immunoblot analysis}

The proteins resolved by 1-dimensional (1D) or 2D PAGE were transferred onto a PVDF membrane (Millipore, Billerica, MA, USA). The membranes were incubated overnight at $4^{\circ} \mathrm{C}$ with antibodies against PRMT1 (1:1,000 dilution, Cell Signaling Technol- ogy, Danvers, MA, USA), PRMT4 (1:5,000, Novus Biology, Centennial, CO, USA), PRMT6 (1:1,000 dilution, Cell Signaling Technology), ASYM24 (1:5,000, Millipore), and $\beta$-actin (1:10,000, Santa Cruz Biotechnology, Santa Cruz, CA, USA). After extensive washing with Tris-buffered saline (TBS), the immunoblot was incubated with peroxidase-conjugated secondary antibody for 1 hour at room temperature and then washed with TBS/0.05\% Tween-20 followed by water. The bound antibodies were visualized by the immunoblotting detection reagents (Millipore). The relative intensities of specific signals were quantified using the Kodak MI imaging system (Kodak, Rochester, NY, USA).

\section{Protein identification by mass spectrometry}

MS analysis of protein samples was performed through a service provided by GENOMINE in Pohang, Korea (www.genomine. com). Spots of interest were manually excised, digested with trypsin (Promega, Madison, WI, USA), mixed with a-cyano-4-hydroxycinnamic acid in $50 \%$ acetonitrile $/ 0.1 \%$ trifluoroacetic acid, and subjected to MALDI-TOF analysis (Ettan MALDI-TOF Pro, GE Healthcare) as described previously [24]. Spectra were collected from 300 shots per spectrum over an $\mathrm{m} / \mathrm{z}$ range of 600 3,000 and calibrated with 2-point internal calibration using trypsin auto-digestion peaks (m/z5842.5099, 2211.1046). A peak list was generated using Flex Analysis 3.0 (Bruker, Billerica, MA, USA). The threshold used for peak picking was as follows: 500 for minimum resolution of monoisotopic mass and 5 for signal to noise. The search program MASCOT, developed by Matrixscience (http://www.matrixscience.com), was used for protein identification by peptide mass fingerprinting (PMF). The following parameters were used for the database search: trypsin as the cleaving enzyme, a maximum of one missed cleavage, iodoacetamide (cysteine) as a complete modification, oxidation (methionine) as a partial modification, monoisotopic masses, and a mass tolerance of 
$\pm 0.1 \mathrm{Da}$. PMF acceptance criteria were based on probability scoring.

\section{In silico analysis of differential expression of mRNAs}

The mRNA expression profiling data sets were obtained from the National Center for Biotechnology Information (NCBI) Gene Expression Omnibus (GEO) database portal (http://www.ncbi.nlm.nih. gov/geo/; accession numbers: GSE10950, GSE113513, GSE41258). Relative expression of mRNAs in the data sets was analyzed by comparing the values between CRC tumor and noncancerous tissues from patients.

\section{RESULTS}

Overexpression of type I protein arginine methyltransferases in colorectal cancer tissues relative to adjacent noncancerous tissues from patients Based on previous reports [11-14], we compared the expression levels of 3 major type I PRMTs (PRMT1, PRMT4, and PRMT6) using Western immunoblots of CRC and adjacent noncancerous tissue extracts from the same patients with stage II and III. As shown in Fig. 2, PRMT1 and PRMT4 expressions increased in most tumor samples relative to the adjacent normal tissues, and these results were consistent with previous reports $[11,13]$. In addition, we recently reported that PRMT6 expression increased by more than 1.5 -fold in $35 \%$ of tumor samples relative to adjacent noncancerous tissues from the same patients [14]. The phenomenon was also reproduced in the present study (Fig. 2).

Comparison of arginine methylation status between CRC and normal tissues from patients

Specific antibodies to detect protein-incorporated ADMA residue were successfully synthesized $[16,23]$. ASYM24 is a representative antibody that we used in the present study [23]. Using the DLD1 and HCT116 cell lines derived from CRC, we further confirmed ASYM24 specificity using type I PRMT inhibitor. Eram et al. [25] have synthesized a highly specific inhibitor (MS023) of type I PRMTs and they showed that MS023 is completely inactive against type II PRMTs, protein lysine methyltransferases, and DNA methyltransferases [25]. DLD1 and HCT116 cells were treated with 3 increasing concentrations of MS023 for 48 hours. While ASYM24 signals at the $27-\mathrm{kDa}$ and $150-\mathrm{kDa}$ molecular masses showed an MS023 concentration-dependent decrease, those between $60 \mathrm{kDa}$ and $90 \mathrm{kDa}$ clearly decreased at the lowest concentration of MS023 in 2 CRC cell lines (Fig. 1A). These results strongly indicated that ASYM24 specifically reacts with protein-incorporated ADMA residues.

To compare the pattern of ADMA-containing proteins between clinical samples, we performed WB with ASYM24 antibody using 10 matched CRC and noncancerous tissues from patients. As shown in Fig. 1A, the pattern of ADMA-containing proteins in CRC tissues was generally similar to that of 2 CRC cell lines, suggesting that 2 biological sources share common substrates [20]. In addition, the levels of arginine modifications between 50 and 100 $\mathrm{kDa}$ are significantly elevated in most tumor samples relative to matched normal samples (Fig. 1B). This phenomenon might be due to overexpression of the 3 type I PRMTs as shown in Fig. 1B and/or their substrate proteins.

\section{Identification of arginine-methylated proteins in HCT116 cells}

Based on the similar pattern of ADMA between CRC cell lines and tissues (Fig. 1), we sought to identify individual ADMA-containing proteins using $2 \mathrm{D}-\mathrm{GE}$ and immunodetection by $\mathrm{WB}$ with
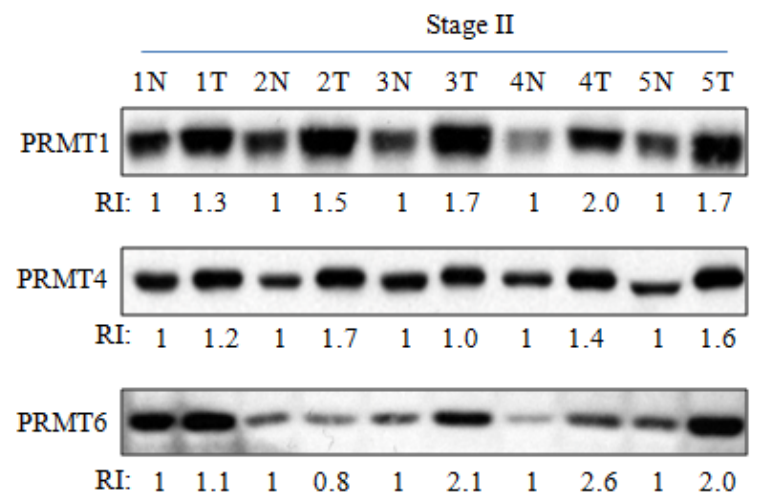

$\beta$-actin
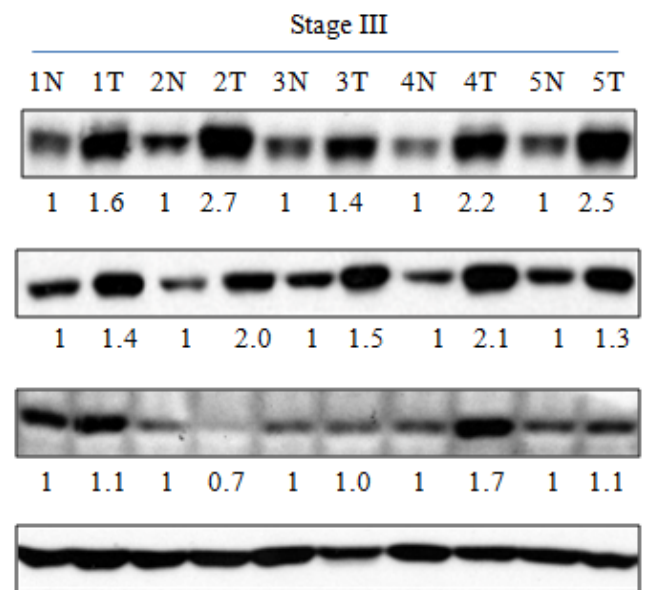

B

Fig. 2. Increased expression of type I protein arginine methyltransferases (PRMTs) in colorectal cancer (CRC) tissues from patients. Tissue lysates $(20 \mu \mathrm{g})$ from CRC and adjacent noncancerous tissues from patients were subjected to Western blot analysis with the respective type I PRMT antibodies was carried out using tissue lysates. Densitometric analysis of 3 major type I PRMTs expression was performed to compare the primary CRC $(\mathrm{T})$ and adjacent noncancerous tissues (N) paired from the same patient with stage II (A) and III (B) CRC. RI, relative intensity. 
the ASYM24 antibody in HCT116 cells. Using HCT116 protein extract, 1D-IEF was performed as described in the Methods section followed by 2D-GE in duplicates. After 2D-GE, one gel was stained with CBB (Fig. 3B) and the other used for immunobloting with ASYM24 (Fig. 3B). There were 891 CBB-stained spots in the 2D gel and 242 2D-WB signals by ASYM24 antibody (Fig. 3C and Supplementary Fig. 1). The pattern of 2D-WB signals was generally correlated with that of $1 \mathrm{D}-\mathrm{WB}$. The major signals between 50 and $100 \mathrm{kDa}$ that were detected by the ASYM24 antibody on $1 \mathrm{D}$ immunoblot (lane 5 in Fig. 1A) were also found in 2D-WB. In addition, the $27 \mathrm{kDa}$ signal on 1D immunoblot of HCT116 cells was detected in the 2D-WB (spot No. 8005 in Fig. 3B).

Using PDQuest software (ver. 8.0), we assigned the signals on $2 \mathrm{D}-\mathrm{WB}$ to the $\mathrm{CBB}$-stained proteins in the 2D polyacrylamide gel and 142 signals were matched (Fig. 3C). Among these spots, we selected 7 CBB-stained spots which showed relatively low protein
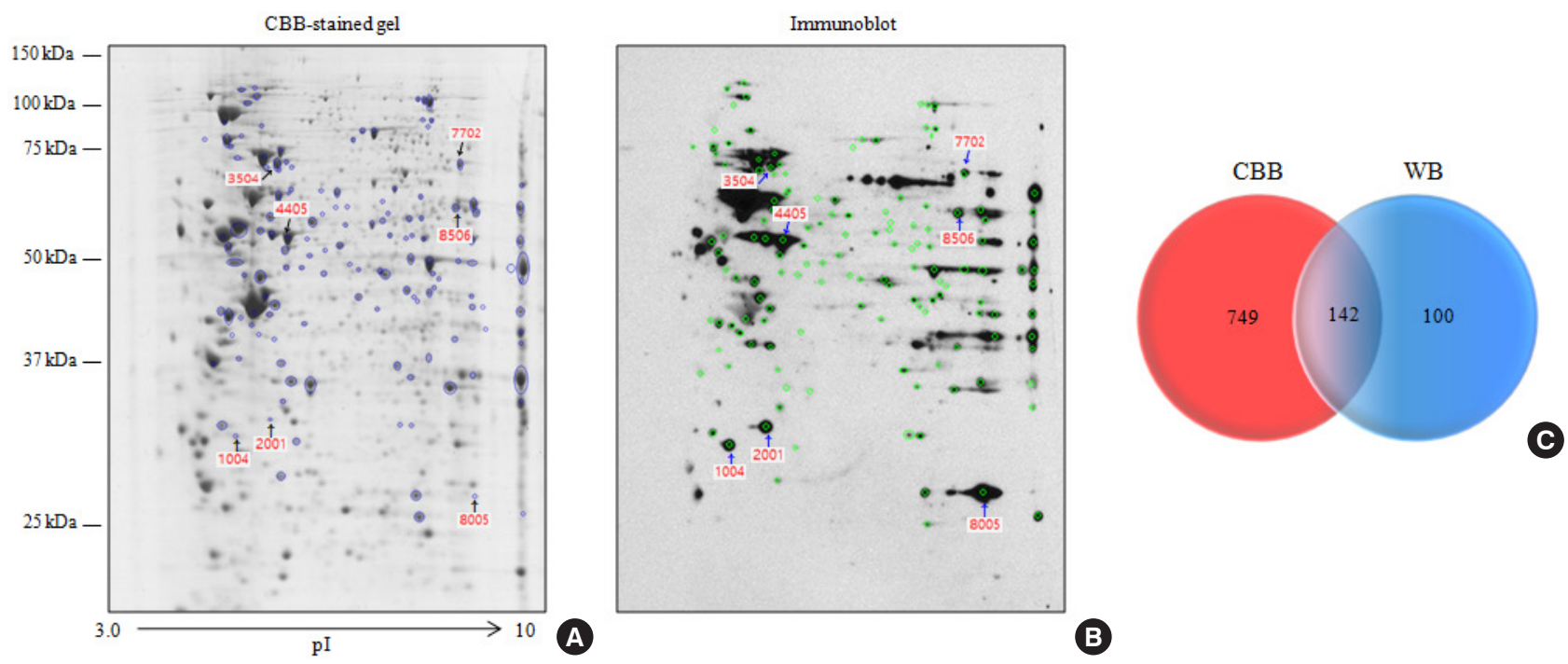

Fig. 3. Two-dimensional spot matching between Coomassie blue (CBB)-stained proteins in polyacrylamide gel and methyl-arginine signals on immunoblot. (A) One gel was stained with CBB. Blue outlines indicate protein spots corresponding to immuno-signals in the panel B. The protein spots used for identification by mass spectrometry are indicated by arrows and numbers. (B) The other gel was transferred to a PVDF membrane (Millipore, Billerica, MA, USA) followed by immunoblotting with the anti-dimethyl-arginine antibody, asymmetric antibody. Immunoblot signals that correlate to protein spots in panel A are indicated with green outlines. Arrows and numbers also indicate immuno-signals corresponding to protein spots in the panel A. (C) The total spot numbers of CBB-stained proteins and immunoblot signals as well as common spot numbers among the 2 images are shown as a Venn diagram. WB, Western blotting.

Table 1. List of arginine-methylated proteins identified by mass spectrometry

\begin{tabular}{|c|c|c|c|c|c|c|c|c|c|}
\hline \multirow{2}{*}{ No. } & \multirow{2}{*}{$\begin{array}{l}\text { Spot } \\
\text { No. }\end{array}$} & \multirow{2}{*}{$\begin{array}{l}\text { Protein } \\
\text { identifier }\end{array}$} & \multirow{2}{*}{$\begin{array}{c}\text { Uniprot } \\
\text { accession } \\
\text { No. }\end{array}$} & \multirow{2}{*}{$\begin{array}{l}\text { Theoretical } \\
\mathrm{pl} / \mathrm{Mw}(\mathrm{kDa})\end{array}$} & \multirow{2}{*}{$\begin{array}{c}\text { Sequence } \\
\text { coverage }(\%) / \\
\text { protein score }^{\mathrm{a}}\end{array}$} & \multirow[b]{2}{*}{ Protein class } & \multicolumn{2}{|c|}{ Spot intensity ${ }^{b}$} & \multirow{2}{*}{$\begin{array}{c}\text { Ratio } \\
\text { (immunoblot/ } \\
\text { CBB) }\end{array}$} \\
\hline & & & & & & & CBB & Immunoblot & \\
\hline 1 & 1004 & MAPRE & Q15691 & $5.02 / 30.15$ & $49 / 152$ & Cytoskeleton & 643.95 & $3,764.59$ & 5.8 \\
\hline 2 & 2001 & GLOD4 & Q9HC38 & $5.40 / 33.53$ & $57 / 194$ & Not classified & 642.56 & $5,547.59$ & 8.6 \\
\hline 3 & 3504 & HSPA8 & P11142 & $5.37 / 71.08$ & $43 / 225$ & Molecular chaperone & $2,064.56$ & $7,853.16$ & 3.8 \\
\hline 4 & 4405 & CK8 & P05787 & $5.52 / 53.52$ & $36 / 117$ & Cytoskeloton & $6,563.26$ & $10,896.36$ & 1.7 \\
\hline 5 & 7702 & TKT & P29401 & $7.90 / 68.43$ & $48 / 293$ & Transferase & 857.24 & $1,051.78$ & 1.2 \\
\hline 6 & 8005 & CACYBP & Q9HB71 & 8.28/26.3 & $46 / 112$ & Ubiquitin conjugation pathway & 152.49 & $15,231.49$ & 99.9 \\
\hline 7 & 8506 & CCT7 & Q99832 & $7.55 / 59.65$ & $48 / 272$ & Component of molecular chaperone & 521.59 & $1,879.95$ & 3.6 \\
\hline
\end{tabular}

$\mathrm{pl}$, isoelectric point; Mw, molecular weight; CBB, Coomassie blue; MAPRE, microtubule-associated protein RP/EB family member 1; GLOD4, glyoxalase domain-containing 4; HSPA8, heat shock cognate 71 kDa protein; CK8, cytokeratin 8; TKT, transketolase; CACYBP, calcyclin-binding protein; CCT7, chaperonin containing T-complex protein 1 subunit 7 eta.

${ }^{a}$ All protein scores corresponding to each protein are significant $(\mathrm{P}<0.005)$. ${ }^{\text {BBoth }} \mathrm{CBB}$-stained protein and 2-dimensional Western blotting spot intensities were analyzed with PDQuest software (Bio-Rad, Hercules, CA, USA). 
staining intensity (spot No. 8005) to high intensity (spot No. 4405), as shown in Table 1. These spots were extracted from the CBB-stained gel and successfully identified by MS (Table 1). Among the identified substrates listed in Table 1, calcyclin-binding protein (CACYBP) [18], chaperonin containing T-complex protein 1 subunit 7 eta (CCT7) [17], heat shock cognate $71 \mathrm{kDa}$ protein 8 (HSPA8) [17], cytokeratin 8 (CK8) [16], and transketolase (TKT) [18] proteins were previously reported as argininemethylated proteins. This indicates that the signals detected in 2D-WB are methyl-arginine specific, and this method can be a useful method to identify substrate proteins for PRMTs. In particular, although CACYBP showed the lowest CBB-staining intensity among the 7 proteins identified, its immunosignal by ASYM24 antibody showed the highest intensity (Table 1), suggesting that CACYBP might be a preferential target for type I PRMTs. However, microtubule-associated protein RP/EB family member 1 (MAPRE1) and glyoxalase domain-containing 4 (GLOD4) were identified as new targets for type I PRMTs in this study.
Comparison of messenger RNA expression of the identified proteins by mass spectrometry between colorectal cancer and noncancerous tissues

Recently, we reported that CRC tissue maintains significantly higher level of symmetric arginine dimethylation compared to matched noncancerous tissue by quantitative analysis [21]. Among the proteins showing elevated symmetric arginine modification, several SDMA-containing proteins were overexpressed in CRC tissues relative to paired noncancerous tissues, indicating that elevated arginine methylation in CRC tissues was mainly due to overexpression of the substrate proteins [21]. Using 3 independent microarray datasets from the NCBI GEO database, we compared the mRNA expression of the 7 methyl-accepting proteins between CRC and adjacent normal tissues. Relative expression of GLOD4 and CK8 genes in the microarray datasets revealed no significant change between the 2 groups or down-regulation in $\mathrm{CRC}$ tissues relative to noncancerous tissues (Supplementary Fig. 2). However, MAPRE1, CCT7, TKT, and 2 splicing variants of
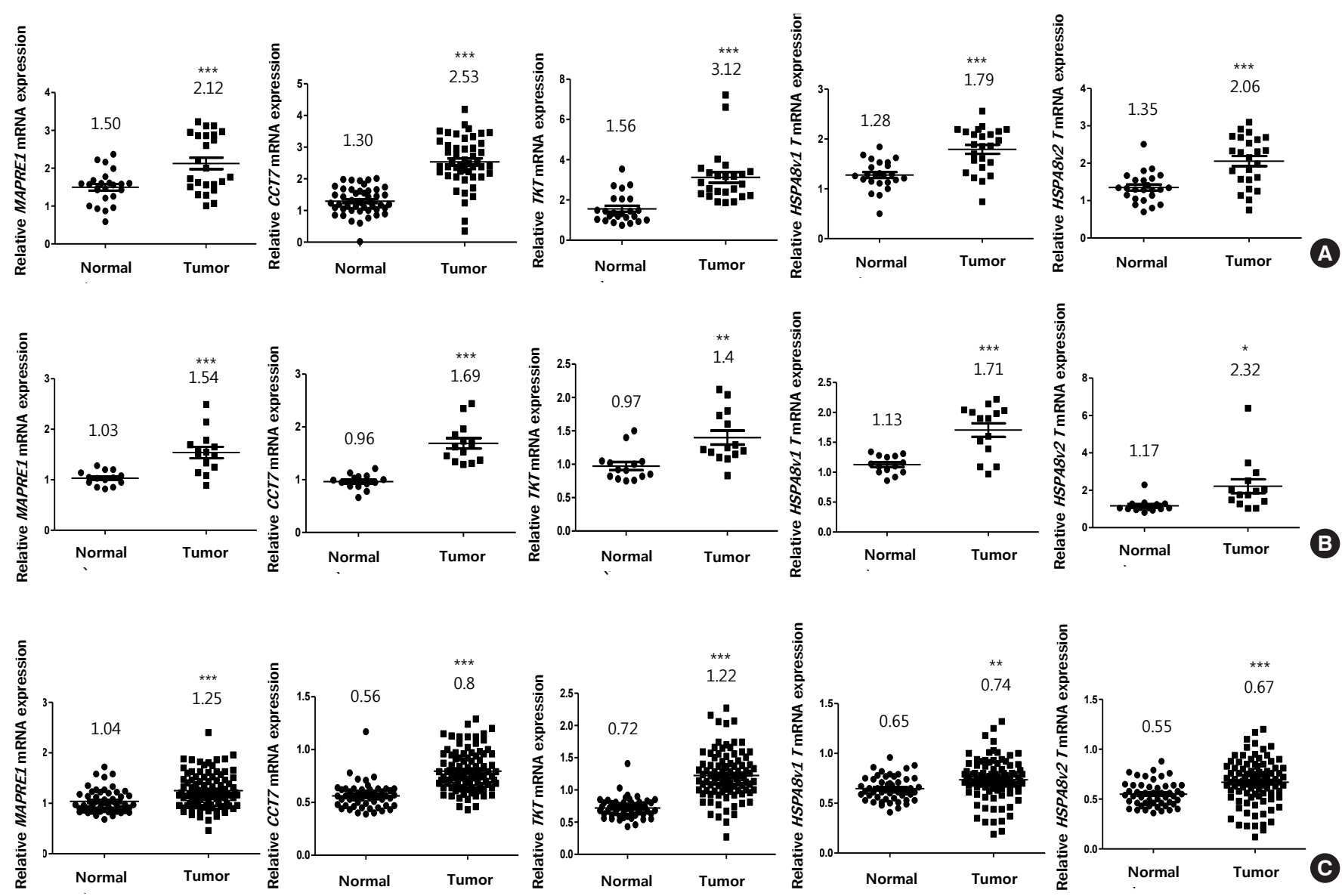

Fig. 4. Overexpression of MAPRE1, CCT7, TKT, and HSPA8 messenger RNAs (mRNAs) in colorectal cancer (CRC) tissues compared to noncancerous tissues via in silico analysis. We analyzed the relative levels of each gene in the adjacent normal group (Normal) and CRC group (Tumor) using 3 independent sets of National Center for Biotechnology Information microarray data. The number of tissue samples for GSE10950 (A) and GSE113513 (B) data sets are 24 and 14, respectively. GSE41258 (C) data set contains 52 noncancerous and 96 CRC tissues. ${ }^{\star} \mathrm{P}<0.05$, ${ }^{* *} \mathrm{P}<0.001,{ }^{* * *} \mathrm{P}<0.0001$. 
HSPA8 (HSPA8 v1 and v2) mRNAs showed statistically significant increase in CRC tumor tissues compared to adjacent normal tissues in all microarray datasets (Fig. 4). While CACYBP mRNA level was also significantly up-regulated in CRC tumor tissues relative to noncancerous tissues in GSE113513 and GSE41258 data sets, it showed no significant change in GSE10950 data set (Supplementary Fig. 2). Therefore, we compared CACYBP level using tissue extracts as shown in Fig. 2 and found that the protein was highly expressed in CRC tissues compared with adjacent normal tissues (Supplementary Fig. 3).

A critical assumption in studying mRNA expression is that it is informative in the prediction of protein expression. However, the degree of correlation between mRNA and protein levels in various biological sources show weakly positive correlation, indicating differences in their regulatory mechanisms [26-28]. Therefore, it should be noted that expression of 7 proteins identified in this study must be further identified using immunohistochemistry between noncancerous and CRC tissues.

\section{DISCUSSION}

In the present study, we found that arginine methylation levels, as well as type I PRMTs, are highly maintained in CRC tissues compared to adjacent noncancerous tissues. These results strongly support that CRC tumors and adjacent noncancerous tissues are a good source for further identification and quantification of arginine methylation in proteins. Recently, we reported a number of MMA, ADMA, and SDMA sites in CRC tissues at the proteome level using immunoenrichment of arginine-methylated peptides combined with high-resolution liquid chromatography-MS/MS $[20,21]$. There was a significant presence of methyl-arginine residues on nucleic acid binding proteins, protein complexes involved in transcription, and enzymes [20, 21]. Among the 7 proteins identified in the present study, HSPA8 and CCT7 were also identified as MMA-containing proteins in CRC tissues [20, 21]. HSPA8 is a chaperone protein that represents a constitutively expressed cognate protein of the HSP70 family. The arginine 469 residue (R469) of HSPA8 is highly conserved among HSP70 family members [29] and the residue is monomethylated by PRMT4 [30] and PRMT7 [29]. R469 monomethylation of the HSPA8 by PRMT7 contributes to the cytoprotective and stress responses [26]. CCT7 is a component of the CCT oligomer, which consists of 8 individual protein subunits, named either $\alpha$ to $\theta$ or 1 to 8 [31]. The CCT oligomer is majorly required for the folding of newly synthesized actin and tubulin molecules [32]. Among 8 members of the CCT complex, only CCT7 has RGG and GRG motifs on its $\mathrm{N}$-terminal region, which are the preferential target motifs for PRMTs $[16,33]$. However, the functional significance of arginine modification of CCT7 in protein folding during CRC tumorigenesis is unclear.

Larsen et al. [18] identified over 8,000 arginine monomethylation sites within 3,300 proteins in human embryonic kidney 293 cells using high-resolution MS and identified methylation of the $\mathrm{R} 162$ residue of CACYBP. Interestingly, CACYBP is a component of the ubiquitin pathway that associates with the Skp1-Cullin1-Fbox complex and is involved in the regulation of Siah-1-induced reduction in $\beta$-catenin protein level [34]. Interestingly, PRMT4, which was increased in CRCs compared with matched normal tissues (Fig. 2A), is a CACYBP-binding partner [35]. In this study, we found that CACYBP is highly methylated compared to its protein level in HCT116 cells (Table 1). Therefore, identification of catalytic enzyme for CACYBP methylation and the effect of arginine methylation of CACYBP on $\beta$-catenin stability should be further identified.

CK8 and TKT are known to contain multiple methyl-arginine sites $[16,18]$. TKT is a rate-limiting enzyme in nonoxidative phase of the pentose phosphate pathway (PPP) [36] and 3 genes have been identified in the human genome encoding TKT isozymes: TKT, TKTL1, and TKTL2 [37]. In CRC, while Langbein et al. [38] reported that there was no difference in TKT mRNA expression between tumor and normal tissues from 54 patients, the mRNA expression retrieved from the Oncomine database showed significant increase in rectal and colon adenocarcinoma compared to noncancerous tissues [39]. In the present study, we also found that TKT mRNA level was significantly elevated in 3 microarray data sets (Fig. 4). The PPP is a very important metabolic pathway that connects with glycolysis and ribose-5-phosphate, and creates reduced nicotinamide adenine dinucleotide phosphate as a major product. Consequently, the PPP plays a pivotal role in helping glycolytic cancer cells to meet their anabolic demands and combat oxidative stress [40]. It is therefore interesting to investigate the effect of TKT arginine methylation on enzymatic activity or interaction with its substrates. CK8 is a member of type II (neutral-basic, CK1-CK8) gene family [41], and the epithelial cytoskeleton is predominantly formed by cytokeratins that are involved in maintaining cell morphology and protecting cells from mechanical and nonmechanical stressors [42]. In CRC, reduced expression of CK8 and CK20 has been associated with epithelial-to-mesenchymal cancer cell transition, which is generally indicative of higher tumor aggressiveness, and decreased patient survival [43]. In accordance with these reports, CK8 mRNA expression revealed significant down-regulation in GSE113513 and GSE41258 data sets (Supplementary Fig. 2).

GLOD4 and MAPRE1 have been identified as novel candidates for type I PRMTs. GLOD4 belongs to the glyoxalase I family and was firstly isolated from hepatocellular carcinoma in humans [44]. MARPE1 is a microtubule protein that binds to the tumor suppressor adenomatous polyposis coli (APC) [45]. While the expression pattern, molecular function, and its role during CRC tumorigenesis of GLOD4 have not yet been addressed, MARPE1 expression is increased in human CRC progression and both genetic and chemically induced models of CRC, consistent with a decrease in APC [46]. In addition, MARPE1 was suggested as a plasma biomarker for early-stage CRC and adenomas, when 
combined with other molecules such as carcinoembryonic antigen and adenylate kinase isoenzyme 1 [47]. Collectively, quantitative analysis of arginine modification among sera from patients with CRC and healthy donors may provide a new biomarker candidate for CRC.

Recent advances in MS methodology provide multiple choices to identify arginine-methylated proteins and specific modification sites. In addition, the development of highly specific antibodies against methylarginines in synthetic peptides has made it possible to enrich all types of arginine-methylated peptides, followed by high-resolution MS analysis. Using these technologies, thousands of arginine-methylated proteins from various biological sources were identified [17-21]. However, the methyl-specific antibodies used to enrich for arginine-methylated motifs could recognize a subset of substrates that would bias the motif description. In the present study, we showed that 2D-GE followed by WB with ASYM24 antibody could successfully identify new substrates for PRMTs in CRC cell line. This method might be useful for direct comparison of PRMTs' substrate level in clinical samples as well as identification of methylarginine-containing proteins.

\section{CONFLICT OF INTEREST}

No potential conflict of interest relevant to this article was reported.

\section{ACKNOWLEDGMENTS}

This research was supported by the National Research Foundation of Korea grant (NRF-2014R1A2A2A01004362) funded by the Korean government (MSIP) and by the Korean Society of Coloproctology Boryung Research Grant 2014.

\section{SUPPLEMENTARY MATERIALS}

Supplementary materials for this study are presented online (available at https://doi.org/10.3393/ac.2020.00899.0128).

\section{REFERENCES}

1. Rawla P, Sunkara T, Barsouk A. Epidemiology of colorectal cancer: incidence, mortality, survival, and risk factors. Prz Gastroenterol 2019;14:89-103.

2. Koveitypour Z, Panahi F, Vakilian M, Peymani M, Seyed Forootan F, Nasr Esfahani MH, et al. Signaling pathways involved in colorectal cancer progression. Cell Biosci 2019;9:97.

3. Sideris M, Papagrigoriadis S. Molecular biomarkers and classification models in the evaluation of the prognosis of colorectal cancer. Anticancer Res 2014;34:2061-8.

4. Bedford MT, Richard S. Arginine methylation an emerging regulator of protein function. Mol Cell 2005;18:263-72.

5. Bedford MT, Clarke SG. Protein arginine methylation in mam- mals: who, what, and why. Mol Cell 2009;33:1-13.

6. Paik WK, Paik DC, Kim S. Historical review: the field of protein methylation. Trends Biochem Sci 2007;32:146-52.

7. Zurita-Lopez CI, Sandberg T, Kelly R, Clarke SG. Human protein arginine methyltransferase 7 (PRMT7) is a type III enzyme forming $\omega$-NG-monomethylated arginine residues. J Biol Chem 2012; 287:7859-70.

8. Aletta JM, Hu JC. Protein arginine methylation in health and disease. Biotechnol Annu Rev 2008;14:203-24.

9. Yang Y, Bedford MT. Protein arginine methyltransferases and cancer. Nat Rev Cancer 2013;13:37-50.

10. Li $\mathrm{X}$, Wang $\mathrm{C}$, Jiang $\mathrm{H}$, Luo $\mathrm{C}$. A patent review of arginine methyltransferase inhibitors (2010-2018). Expert Opin Ther Pat 2019; 29:97-114.

11. Mathioudaki K, Papadokostopoulou A, Scorilas A, Xynopoulos D, Agnanti N, Talieri M. The PRMT1 gene expression pattern in colon cancer. Br J Cancer 2008;99:2094-9.

12. Ou CY, LaBonte MJ, Manegold PC, So AY, Ianculescu I, Gerke DS, et al. A coactivator role of CARM1 in the dysregulation of $\beta$-catenin activity in colorectal cancer cell growth and gene expression. Mol Cancer Res 2011;9:660-70.

13. Kim YR, Lee BK, Park RY, Nguyen NT, Bae JA, Kwon DD, et al. Differential CARM1 expression in prostate and colorectal cancers. BMC Cancer 2010;10:197.

14. Lim Y, Yu S, Yun JA, Do IG, Cho L, Kim YH, et al. The prognostic significance of protein arginine methyltransferase 6 expression in colon cancer. Oncotarget 2017;9:9010-20.

15. Peng C, Wong CC. The story of protein arginine methylation: characterization, regulation, and function. Expert Rev Proteomics 2017;14:157-70.

16. Guo A, Gu H, Zhou J, Mulhern D, Wang Y, Lee KA, et al. Immunoaffinity enrichment and mass spectrometry analysis of protein methylation. Mol Cell Proteomics 2014;13:372-87.

17. Geoghegan V, Guo A, Trudgian D, Thomas B, Acuto O. Comprehensive identification of arginine methylation in primary $\mathrm{T}$ cells reveals regulatory roles in cell signalling. Nat Commun 2015;6: 6758.

18. Larsen SC, Sylvestersen KB, Mund A, Lyon D, Mullari M, Madsen MV, et al. Proteome-wide analysis of arginine monomethylation reveals widespread occurrence in human cells. Sci Signal 2016;9:rs9.

19. Musiani D, Bok J, Massignani E, Wu L, Tabaglio T, Ippolito MR, et al. Proteomics profiling of arginine methylation defines PRMT5 substrate specificity. Sci Signal 2019;12:eaat8388.

20. Lim Y, Lee JY, Ha SJ, Yu S, Shin JK, Kim HC. Proteome-wide identification of arginine methylation in colorectal cancer tissues from patients. Proteome Sci 2020;18:6.

21. Lim Y, Park YE, Ha S, Lee JE, Kim HC. A comprehensive analysis of symmetric arginine dimethylation in colorectal cancer tissues using immunoaffinity enrichment and mass spectrometry. Proteomics 2020 May 28 [Epub]. https://doi.org/10.1002/pmic.201900367.

22. Thandapani P, O'Connor TR, Bailey TL, Richard S. Defining the 
RGG/RG motif. Mol Cell 2013;50:613-23.

23. Boisvert FM, Côté J, Boulanger MC, Richard S. A proteomic analysis of arginine-methylated protein complexes. Mol Cell Proteomics 2003;2:1319-30.

24. Fernandez J, Gharahdaghi F, Mische SM. Routine identification of proteins from sodium dodecyl sulfate-polyacrylamide gel electrophoresis (SDS-PAGE) gels or polyvinyl difluoride membranes using matrix assisted laser desorption/ionization-time of flightmass spectrometry (MALDI-TOF-MS). Electrophoresis 1998;19: 1036-45.

25. Eram MS, Shen Y, Szewczyk M, Wu H, Senisterra G, Li F, et al. A Potent, Selective, and cell-active inhibitor of human type i protein arginine methyltransferases. ACS Chem Biol 2016;11:772-81.

26. Chen G, Gharib TG, Huang CC, Taylor JM, Misek DE, Kardia SL, et al. Discordant protein and mRNA expression in lung adenocarcinomas. Mol Cell Proteomics 2002;1:304-13.

27. Washburn MP, Koller A, Oshiro G, Ulaszek RR, Plouffe D, Deciu $\mathrm{C}$, et al. Protein pathway and complex clustering of correlated $\mathrm{mRNA}$ and protein expression analyses in Saccharomyces cerevisiae. Proc Natl Acad Sci U S A 2003;100:3107-12.

28. Guo Y, Xiao P, Lei S, Deng F, Xiao GG, Liu Y, et al. How is mRNA expression predictive for protein expression? A correlation study on human circulating monocytes. Acta Biochim Biophys Sin (Shanghai) 2008;40:426-36.

29. Szewczyk MM, Ishikawa Y, Organ S, Sakai N, Li F, Halabelian L, et al. Pharmacological inhibition of PRMT7 links arginine monomethylation to the cellular stress response. Nat Commun 2020; 11:2396.

30. Gao WW, Xiao RQ, Peng BL, Xu HT, Shen HF, Huang MF, et al. Arginine methylation of HSP70 regulates retinoid acid-mediated RAR $\beta 2$ gene activation. Proc Natl Acad Sci U S A 2015;112:E332736.

31. Valpuesta JM, Martín-Benito J, Gómez-Puertas P, Carrascosa JL, Willison KR. Structure and function of a protein folding machine: the eukaryotic cytosolic chaperonin CCT. FEBS Lett 2002; 529:11-6.

32. Brackley KI, Grantham J. Activities of the chaperonin containing TCP-1 (CCT): implications for cell cycle progression and cytoskeletal organisation. Cell Stress Chaperones 2009;14:23-31.

33. Lim Y, Hong E, Kwon D, Lee E. Proteomic identification and comparative analysis of asymmetrically arginine-methylated proteins in immortalized, young and senescent cells. Electrophoresis 2010;31:3823-33.

34. Matsuzawa SI, Reed JC. Siah-1, SIP, and Ebi collaborate in a novel pathway for beta-catenin degradation linked to $\mathrm{p} 53$ responses. Mol Cell 2001;7:915-26.

35. Taipale M, Tucker G, Peng J, Krykbaeva I, Lin ZY, Larsen B, et al. A quantitative chaperone interaction network reveals the architecture of cellular protein homeostasis pathways. Cell 2014;158:43448.

36. Horecker BL. The pentose phosphate pathway. J Biol Chem 2002;277:47965-71.

37. Coy JF, Dübel S, Kioschis P, Thomas K, Micklem G, Delius H, et al. Molecular cloning of tissue-specific transcripts of a transketolase-related gene: implications for the evolution of new vertebrate genes. Genomics 1996;32:309-16.

38. Langbein S, Zerilli M, Zur Hausen A, Staiger W, Rensch-Boschert $\mathrm{K}$, Lukan N, et al. Expression of transketolase TKTL1 predicts colon and urothelial cancer patient survival: Warburg effect reinterpreted. Br J Cancer 2006;94:578-85.

39. Xu IM, Lai RK, Lin SH, Tse AP, Chiu DK, Koh HY, et al. Transketolase counteracts oxidative stress to drive cancer development. Proc Natl Acad Sci U S A 2016;113:E725-34.

40. Patra KC, Hay N. The pentose phosphate pathway and cancer. Trends Biochem Sci 2014;39:347-54.

41. Hesse M, Magin TM, Weber K. Genes for intermediate filament proteins and the draft sequence of the human genome: novel keratin genes and a surprisingly high number of pseudogenes related to keratin genes 8 and 18. J Cell Sci 2001;114(Pt 14):2569-75.

42. Karantza V. Keratins in health and cancer: more than mere epithelial cell markers. Oncogene 2011;30:127-38.

43. Makino T, Yamasaki M, Takeno A, Shirakawa M, Miyata H, Takiguchi S, et al. Cytokeratins 18 and 8 are poor prognostic markers in patients with squamous cell carcinoma of the oesophagus. $\mathrm{Br} \mathrm{J}$ Cancer 2009;101:1298-306.

44. Qin WX, Wan F, Sun FY, Zhang PP, Han LW, Huang Y, et al. Cloning and characterization of a novel gene (C17orf25) from the deletion region on chromosome 17p13.3 in hepatocelular carcinoma. Cell Res 2001;11:209-16.

45. Su LK, Burrell M, Hill DE, Gyuris J, Brent R, Wiltshire R, et al. APC binds to the novel protein EB1. Cancer Res 1995;55:2972-7.

46. Stypula-Cyrus Y, Mutyal NN, Dela Cruz M, Kunte DP, Radosevich AJ, Wali R, et al. End-binding protein 1 (EB1) up-regulation is an early event in colorectal carcinogenesis. FEBS Lett 2014;588: 829-35.

47. Taguchi A, Rho JH, Yan Q, Zhang Y, Zhao Y, Xu H, et al. MAPRE1 as a plasma biomarker for early-stage colorectal cancer and adenomas. Cancer Prev Res (Phila) 2015;8:1112-9. 

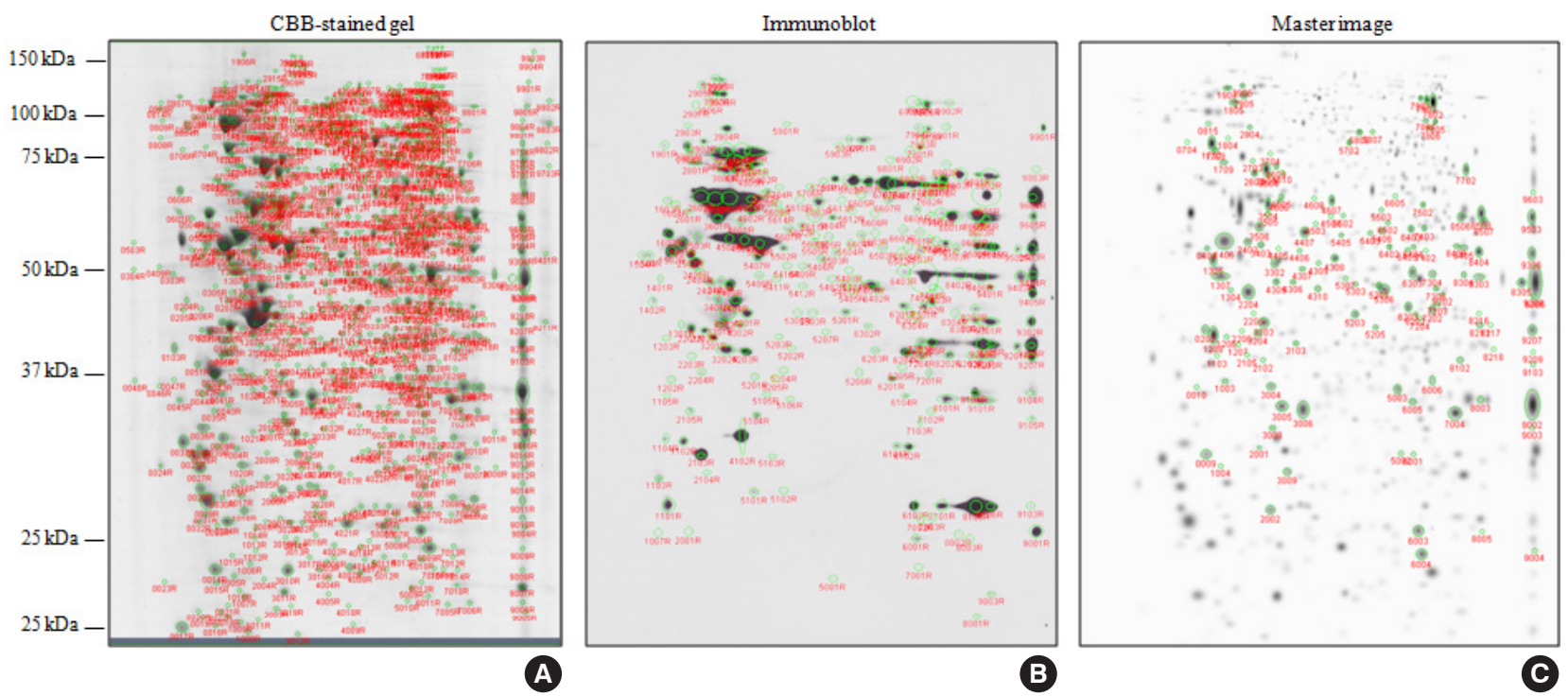

Supplementary Fig. 1. Spot detection and master gel assignment. The total number of Coomassie blue (CBB)-stained protein spots (A) and corresponding 2-dimensional Western blotting (2D-WB) signals (B) was analyzed by PDQuest software (Bio-Rad, Hercules, CA, USA). The CBB-stained gel was selected as the master gel (C) that was subsequently used for spot matching of 2D-WB image. 


\section{Coloproctology Yongchul Lim, et al.}
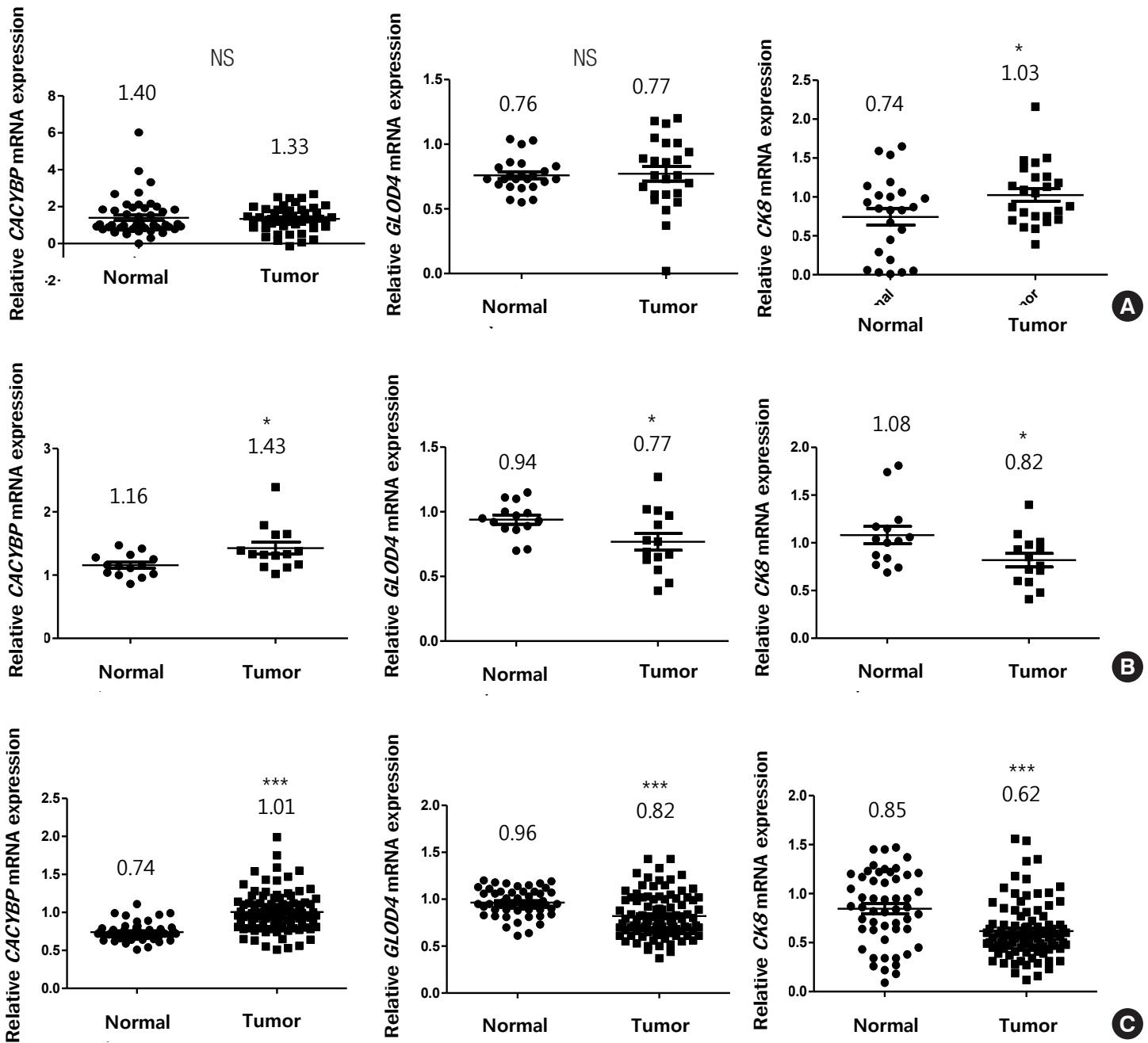

Supplementary Fig. 2. Expression of CACYBP, GLOD4, and CK8 messenger RNAs (mRNAs) between colorectal cancer and noncancerous tissues. GSE10950 (A) and GSE113513 (B) and GSE41258 (C) data sets were used to analyze each mRNA level. ${ }^{*} \mathrm{P}<0.05$, ${ }^{* * *} \mathrm{P}<0.0001$. NS, not significant. 


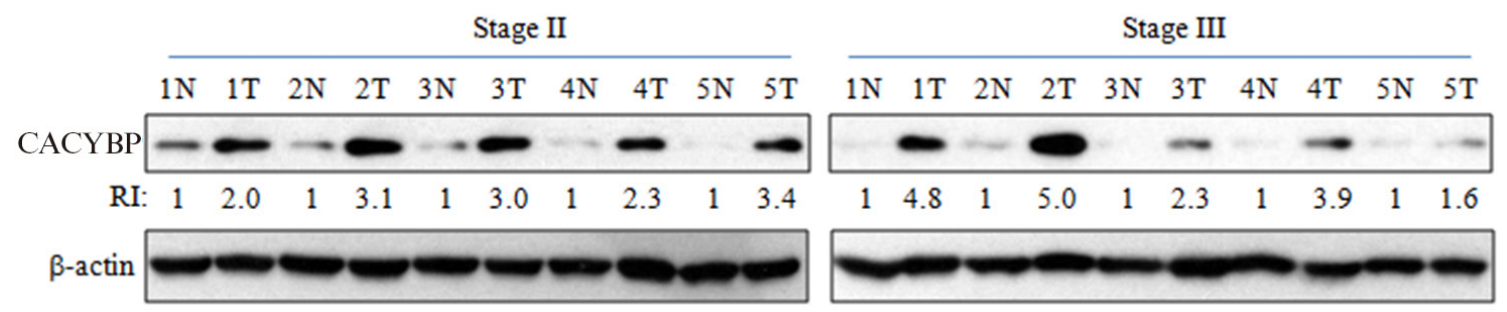

Supplementary Fig. 3. Increased expression of calcyclin-binding protein (CACYBP) in 10 colorectal cancer (CRC) tissues relative to adjacent normal tissues from patients. Densitometric analysis was carried out to compare the primary CRC (T) and adjacent normal tissues (N) paired from the same patient with stage II and III CRC. RI, relative intensity. 